\title{
Significance of the Thalamic Reticular Nucleus GABAergic Neurons in Normal and Pathological Activity of the Brain
}

\author{
Zakaria I. Nanobashvili, Arkadi G. Surmava, Irine G. Bilanishvili, Maia G. Barbaqadze, \\ Magda D. Mariamidze, Nadejda A. Khizanishvili \\ Department of Neurophysiology, Ivane Beritashvili Experimental BioMedicine Centre, Tbilisi, Georgia \\ Email: www.besarionsaba@yahoo.com
}

Received May 28, 2012; revised June 13, 2012; accepted June 22, 2012

\begin{abstract}
The relationship between neurons of the thalamic reticular nucleus (TRN) and relay neurons of the thalamic nuclei was studied. Activation of the TRN neurons was shown to abate activity of relay neurons. This evidence was obtained by stimulation of the TRN and the neocortex and, by introduction of small doses of nembutal as well. Suppression of the relay neuronal activity by the TRN neurons is supposed to occur monosynaptically. It has been also revealed that neuronal activity of the TRN enhances in a clonic phase of seizures generated by stimulation of the hippocampus and as soon as the electroencephalographic seizure reactions disappear. The suppression of limbic motor seizures is obviously related to the process of potentiation in GABAergic synapses of thalamocortical networks. Thus, stimulation of the TRN appears to be a rather valuable methodical tool that can open up prospects in the development of new "anticonvulsive" strategies in the treated of temporal lobe epilepsy.
\end{abstract}

Keywords: Thalamic Reticular Nucleus; Relay Neurons; Seizure Activity; Potentiation; Cats

\section{Introduction}

At present, a number of forms of epileptic attacks are intractable (not sensitive to pharmacological treatment). The search for alternative possibilities for therapy of such disorders motivated ones to study such "antiseizure" approaches as electrical stimulation of afferent nerves and/ or profound structures of the brain. Stimulations of the cerebellum [1], locus coeruleus [2], solitary tract nucleus [3], thalamic nuclei [4], as well as of the n.vagus [5], were tested as influences potentially capable of blocking seizure attacks in humans and experimentally evoked epileptiform discharges in animals. The results of using such methods were, however, contradictory. This is due to insufficient knowledge of the functional organization of the structures, disorders in which result in the development of epilepsy, and different forms and models of epilepsy used in the above-cites studies. This is why structures whose stimulation is capable of inducing an antiseizure effect by preventing initiation and/or spreading of epileptiform reactions have still not been adequately identified.

Several studies have indicated that thalamic nuclei are involved in seizure development and expression [6]. For example, it is well-established that thalamocortical systems participate in the generation of pathological rhythms with 3- to 4-Hz frequency, i.e., spike and wave discharge activity of generalized absence epilepsy. Neurons in the thalamic reticular nucleus (TRN) are involved in the mechanisms of synchronization and generation of this type of seizure activity [7-9]. The TRN forms a shell around the dorsal thalamus and consists of GABAergic neurons [10], which provide a strong inhibitory input on thalamic relay cells [11]. All axons connecting thalamic nuclei and cortical areas in both directions send collaterals to TRN neurons. TRN neurons are activated by thalamocortical and corticothalamic glutamatergic axons and axonal collaterals of thalamic or cortical cells, providing a feedback or feed-forward inhibition, respectively, for thalamic relay neurons [12].

Despite its strategic position in thalamocirtical neural circuity, it is unknown whether TRN is involved in the generalization and expression of limbic seizures. The main objectives were twofold: first, to determine the relations between neurons of the TRN and relay nuclei of the thalamus; because our recent data [13] provide the first evidence that stimulation of TRN can act to suppress limbic motor seizures, second objective was to explore a possible mechanism of blocking the limbic motor seizure reactions induced by activation of the TRN.

\section{Methods}

Experiments were carried out on adult cats $(\mathrm{n}=17)$. Un- 
der ether anesthesia craniotomy was performed and steel bipolar electrodes with factory insulation were used to stimulate TRN or the neocortex. TRN and neocortex was stimulated by single or short series of stimuli. After insertion of the electrodes the administration of ether was stopped and the animals were immobilized by intravenous injection of tubocurarine and artificially ventilated. The experiments began 1.0 - $1.5 \mathrm{~h}$ after administration of ether had ceased. Small doses of nembutal (5 to $8 \mathrm{mg} / \mathrm{kg}$ ) were additionally injected when necessary. All wound surfaces were periodically treated with a $2 \%$ lidocaine solution. Unit responses both from thalamic ventrolateral nucleus (VL) and lateral geniculate body (LGB) and from TRN were derived by glass (tip diameter 1.0 to 1.5 $\mu \mathrm{m}$; resistance about $8 \pm 1,5-2 \mathrm{M} \Omega$ ) or metal microelectrodes (tipdiameter 10 to $15 \mu \mathrm{m}$, with a resistance of between 15 and $40 \mathrm{M} \Omega$. The glass microelectrodes werefilled with potassium or sodium citrate solution (3M). The microelectrodes were directed stereotaxically as they were passed through the neocortex. In some experiments unit activity was recorded simultaneously from TRN and VL or lateral geniculate body (LGB). In the latter case the microelectrode was inserted into TRN at an angle.

Simultaneous recordings were made expracellularly in the VL and TRN.

In experiments, with an aim to record the TRN neuronal activity, the surface of the anterior suprasylvian gyrus and the white matter were removed by suction until exposing the head of the caudate nucleus. The microelectrodes were then lowered through the head of the caudate nucleus in order to reach the rostral section of the TRN. Pentobarbital, in a dose of $0.5-12 \mathrm{mg} / \mathrm{kg}$, was injected intravenously. Relay units of the VL and/or LGB were identified by generation of antidromic spikes after stimulation of the neocortex; these spikes were characterized by a stable latency and reproduced high (300 $400 \mathrm{~Hz}$ ) stimulation frequencies. In some experiments, we used a multilevel amplitude discriminator, which allowed us to separately record spike activity by a single microelectrode from a few single neurons. The location of the insulated tips of the stimulating electrodes was also established in frontal sections.

\section{Data Analysis}

Data analysis was performed with GNU PSPP software version 0.6.2 using the methods of descriptional statistics, paired 2 -tailed t-test $(\alpha<0.05)$ and corellation computing (Pearson r).

\section{Results}

\subsection{Responses of Neurons of Thalamic Relay Nuclei to Stimulation of TRN}

Experiments were carried out on adult cats $(n=3)$. Ac- tivity of 67 VL neurons (14 intracellularly, three quasiintracellularly, the rest extracellularly) and LGB neurons (nine intracellularly, the rest extracellularly) was studied. Under the influence of stimulation of TRN 39\% of the 67 VL neurones were inhibited, $31 \%$ were excited, and the remainder did not respond to stimulation. The single stimulation of TRN led to the appearance of inhibitory postsynaptic potentials (IPSPs) of varied duration (80 to $400 \mathrm{~ms}$ ) and with different latent periods (1 - 2 to $12 \mathrm{~ms}$ ) in LGB neurons. Similar results were obtained when unit activity was recorded in VL. The IPSP arising in VL and LGB neurons in response to single stimulation of TRN was sometimes repeated several times and became, as it were, rhythmic in character, Figure 1.

\subsection{Unit Activity of TRN and Relay Nuclei Evoked by Cortical Stimulation}

Experiments were carried out on adult cats $(n=4)$. Activity of 78 pairs of TRN and VL neurons and of 39 pairs of TRN and LGB neurons was studied in this series of experiments. In the first case reciprocity between TRN and VL neurons was observed in 42 pairs $(53.8 \%)$, in the second case (TRN and LGB) in 19 pairs (48.7\%). In 16 TRN-VL neuron pairs and in 8 TRN-LGB neuron pairs no visible signs of interaction were found. 13 TRN-VL neuron pairs and seven TRN-LGB neuron pairs were simultaneously excited, whereas activity of seven TRNVL and five TRN-LGB neuron pairs was simultaneously inhibited.

The interaction of 21 pairs of TRN and VL neurons in respond to different frequency cortical stimulation is shown in Figure 2.

When single and low-frequency afferent or cortical stimulation (in most cases cortical stimulation was used, as it is know [14] that such stimulation is highly effective for activating TRN neurons) was used several types of interaction between neurons of TRN and the thalamic relay nuclei were found. First, single cortical stimulation evoked the appearance of high-frequency repeated grouped discharges in the TRN neuron, whereas it caused inhibition of discharges in the VL neuron. Second, the lowfrequency cortical stimulation usually led to activation of the TRN neuron and to tonic inhibition of activity of the VL neuron.

\subsection{Effect of Pentobarbital on Unit Activity of TRN and VL Neurons}

Experiments were carried out on adult cats $(n=3)$. Responses of single units in the TRN and VL nuclei were studied in aucte experiments on curarized cats before and after intravenous injection of small $(5-8 \mathrm{mg} / \mathrm{kg})$ doses of pentobarbital, with simultaneous derivation of activity by two microelectrods, Figure 3. 
a

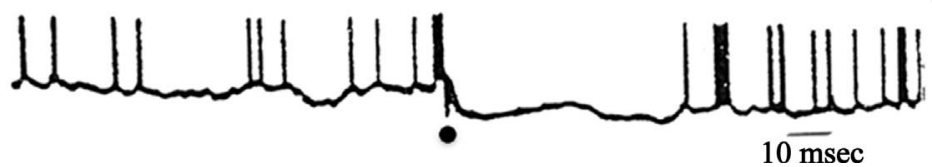

b

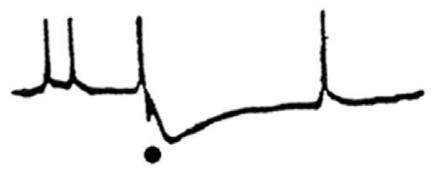

d

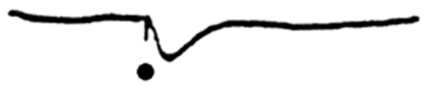

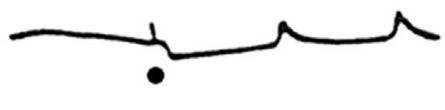

e

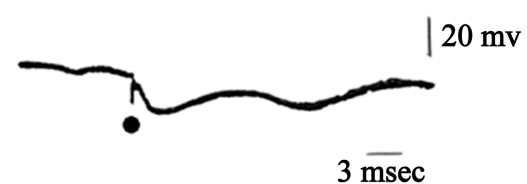

Figure 1. Effects of stimulation of TRN on unit activity in thalamic relay nuclei. (a), (c)—lateral geniculate body; (b), (d), T-ventrolateral nucleus. Dots indicate times of TRN stimulation.

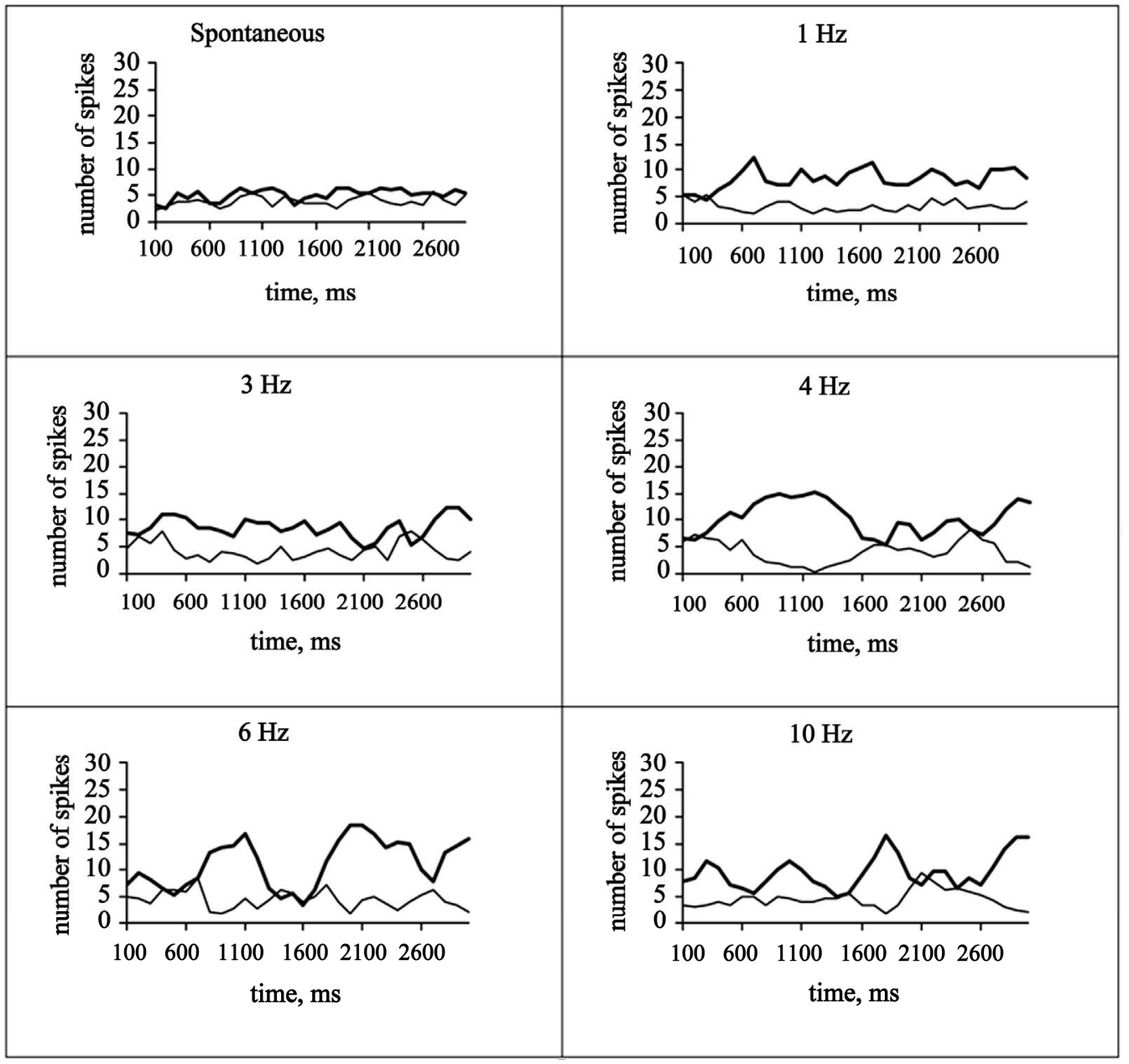

Figure 2. Correlation coefficients, r (Pearson)—spontaneous: 0.2901; 1 Hz: $-0.4670 ; 3$ Hz: $-0.3011 ; 4$ Hz: $-0.7678 ; 6$ Hz: $-0.5616 ; 10 \mathrm{~Hz}$ : $-\mathbf{0 . 5 7 0 2}$. Solid lines-TRN, thin lines-VL. 


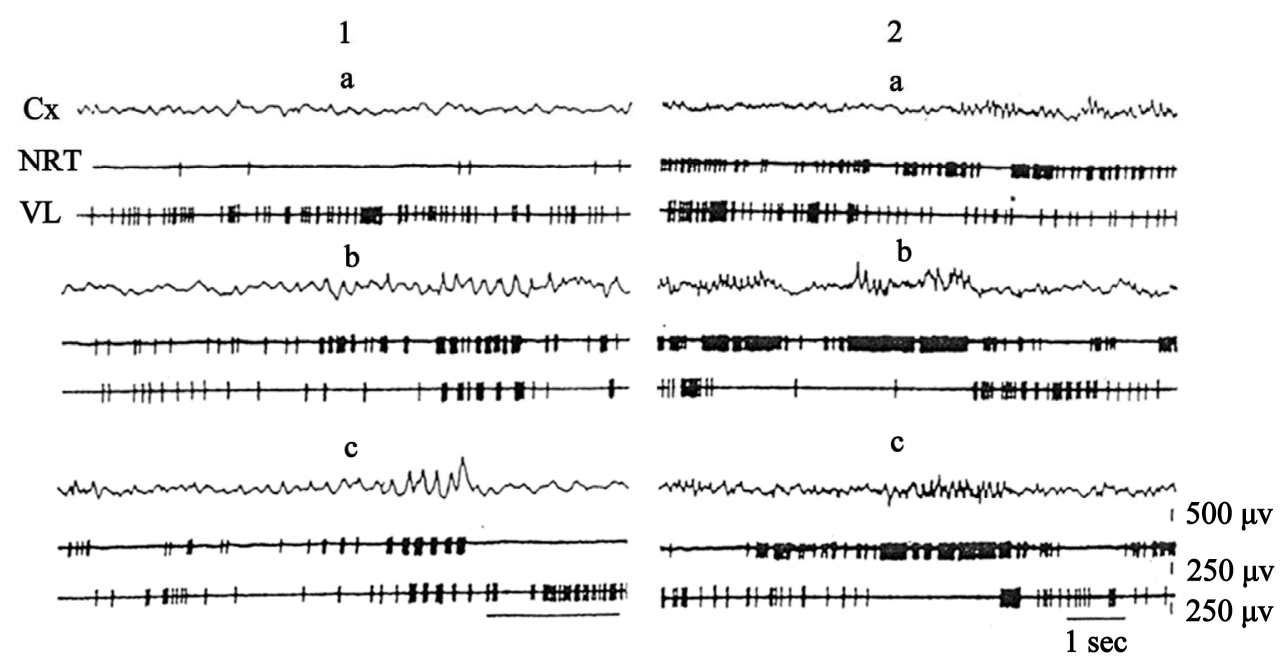

Figure 3. Activity of TRN and VL neurons before (1, 2-different pairs) and after injection of pentobarbital in doses of 8 $\mathrm{mg} / \mathrm{kg}$. Cx-electrical activity of neocortex.
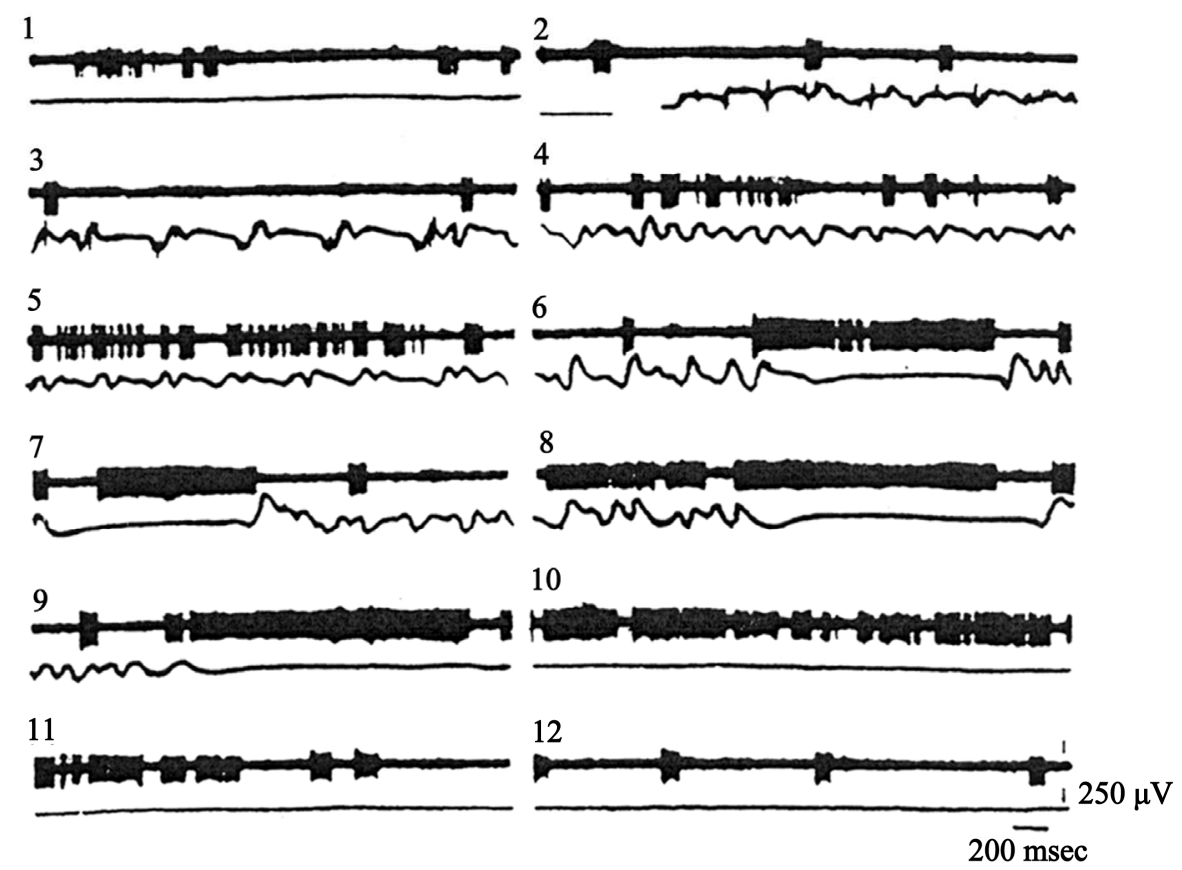

Figure 4. Relationships between the neocortical (lower traces) epileptic activity and spike discharges of the TRN neuron (upper traces) following the high-frequency electrical stimulation (2) of hippocampus $(50 \mu \mathrm{A}, 60 \mathrm{~Hz}, 10 \mathrm{sec})$. 1, spontaneous activity; 2 - 9, evoked seizure discharges; 9 - 12, cessation of epileptic activity.

The study of spontaneous activity of TRN and VL neurons showed desynchronisation of the EEG accompanied by periods of asynchronous activation. The mean spontaneous discharge frequency of TRN neurons was a little higher than that of VL neurons, Figure 3 (a1, a2). The character of spontaneous unit activity in these nuclei changed qualitatively after intravenous injection of small doses $(5-8 \mathrm{mg} / \mathrm{kg})$ of pentobarbital. After intravenous injection of pentobarbital and the appearance of barbiturate spindles on the electroencephalogram (EEG), the low-frequency asynchronous discharges on TRN neuron which were observed before injection of pentobarbital were replaced by high-frequency grouped discharges (52.5\% of 40 neurons). Meanwhile the asynchronous, relatively high-frequency activity of a VL neuron also acquired the character of grouped discharges $(66.5 \%$ of 40 neurons). With the development of relatively well marked spindles excitation of the TRN neuron coincided with inhibition of activity of the VL neuron, Figure 3 ( $1 \mathrm{~b}$, $1 c)$. When activity of other pairs of cells in these nuclei 
was recorded, other types of interaction between TRN and VL neurons were also found. For example, after injection of pentobarbital discharges of the TRN neuron became tonic in character, i.e., throughout the period of recording of spindles - the TRN neuron generated an almost continuous high-frequency spike discharge (30\% of 40 neurons). Activity of the VL neuron showed opposite changes during this period ( $30 \%$ of 40 neurons). In particular, during the appearance of the spindles in the EEG the VL neuron still generated low-frequency regular discharges. Subsequently, the discharges of the VL neuron was almost completely blocked during the periods of continuous excitation of the TRN and of wellmarked spindles in the EEG, Figure 3 (2b, 2c).

\subsection{Involvement of TRN in the Control of Convulsive Activity}

Experiments were carried out on adult cats $(\mathrm{n}=3)$. Hippocampal induced epileptiform activity may be a more suitable model for the epilepsy studies because of its prolonged and progressive development period without severe clinical manifestations and with only focal electrical discharges in evidence as well as with development of epilepsy and secondary generalization [15]. The TRN neurons, during the hippocampal generalized convulsive seizures, were classified into one of the three types, according to their oscillatory properties. Type 1 neurones (23 units out of $120 ; 19.1 \%$ ) were those, in which the spontaneous high-frequency burst discharges density dropped after the onset of the hippocampal evoked epileptic activity. Type 2 neurons (45 units out of 120;37.5\%) were those, in which the activity was significantly enhanced during the silence period of the clonic phases and at the end of seizure episodes. Type 3 neurons (52 units out of $120 ; 43.3 \%$ ) were those, in which discharges were in a close time relation with the EEG self-sustained seizure activity after the cessation of the convulsive activity si multaneous cessation of the TRN neurons seizure discharges was observed. The activity of TRN neurons was significantly enhanced during the silence within the seizure episodes.

\subsection{A Possible Mechanism of Blocking of Limbic Motor Seizure Reactions Induced by Activation of the TRN}

Recently, we showed that the development of kindling induced by hippocampal stimulation can be blocked by simultaneous stimulation of the TRN. The latter stimulation was capable of blocking generalization of the seizures when two different paradigms of kindling were used. Thus, it is believed that the inhibitory influence of stimulation of the TRN does not depend on the type of stimulation of the hippocampus. When discussing other possibilities, we postulated that blocking of the seizure reactions induced by stimulation of the thalamic TRN can result from potentiation of the activity of GABAergic inhibitory neurons of the latter nucleus.

Experiments were carried out on adult cats $(n=4)$. We studied plastic changes in the activity of VL thalamic neurons after stimulations of the TRN. Those neurons (VL) whose activity was inhibited after single TRN stimulations were examined.

Testing was performed in the following way. First, we applied single-pulse stimulations of the TRN, which evoked clear suppresion of the background activity of the VL neurons (Figure 5(b)). Then, the intensity of TRN stimulation was weakened to such a level where single stimulation of the nucleus exerted no noticeable effect on the frequency of background spiking of the VL neuron (Figure 5(c)). Then, the TRN was subjected five times to serial stimulations with the above amplitude of stimuli (Figure 5(d),1-3) — subthreshold at single stimulations. A separate stimulus series was $10 \mathrm{sec}$ long, and these series were separated by 1-min-long intervals. After sach a high-frequency conditioning, single test stimuli of the same amplitude were applied to the TRN (Figure 5(e)). It was found that in most examined neurons relatively high-frequency stimulations of the TRN with the stimulus intensity, which could not provide inhibition of the background activity of VL neurons after single-pulse stimulation, rather effectively suppressed background spiking of the VL units under study. Single-pulse stimulations of this nucleus, which were earlier ineffective, began to significantly suppress the spike activity of the studied VL neurons.

Among 112 examined neurons of the VL, the effects of facilitation of the inhibitory reactions induced by TRN stimulation were preserved for a rather long time interval in 97 units $(86.6 \%)$. The time segment where potentialtion of inhibition was obvious varied in different VL neurons from 5 to $35 \mathrm{~min}$, and in 15 neurons was this effect weak or absent (Figure 6).

Intra- or quasi-intracellular recordings from relay neurons of the VL showed that inhibitory postsynaptic potentials (IPSPs) evoked in these neurons by single stimulations of the TRN are siglificantly facilitated both in the course of conditioning tetanic stimulation of the above nucleus by high-frequency series and for a rather long time after such stimulation. Single stimulation of the TRN with a near-threshold intensity evoked low-amplitude IPSPs in the studied neurons, and their background spike activity was suppressed for 200 to $400 \mathrm{msec}$ (Figure 7(a)). Conditioning tetanic stimulation of the TRN was performed by application of 10 series of stimuli (10 stimuli in each; $50 \mathrm{~Hz}$; interseries intervals $10 \mathrm{sec}$ ). Such high-frequency series evoked IPSPs, whose amplitude 
a

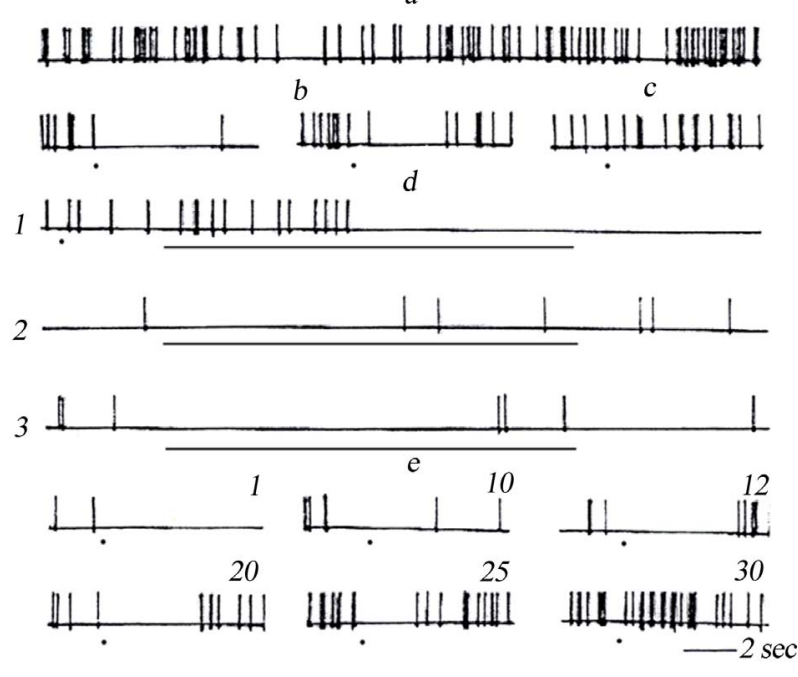

Figure 5. Suppression of the background activity (BA) of a neuron of the VL evoked by tetanic stimulation of the TRN. (a) BA of the VL neuron; b) inhibition of BA of this neuron evoked by single stimulation of the TRN; (c) absence of inhibition of BA after single weaker (subthreshold) stimulation of the TRN; (d) suppression of BA evoked by rhythmic $\left(50 \mathrm{sec}^{-1}\right)$ stimulation of the TRN with stimuli of the same intensity as in panel $\mathrm{C}$ (subthreshold at single stimulations). 1-3 are effects of consecutive rhythmic stimulations of the TRN (the 1st, 3rd, and 5th, respectively) with the intensity subthreshold at single stimulation; (e) Effects of single stimulations of the TRN with stimuli of the same intensity as in panel (c) (earlier subthreshold) with different time intervals (shown below the traces, min, after tetanic stimulation of the TRN (d). Moments of single stimulations of the TRN are shown in panels (b), (c), and $E$ by points; the duration of rhythmic stimulation in panel (d) is shown by a bar below the traces.

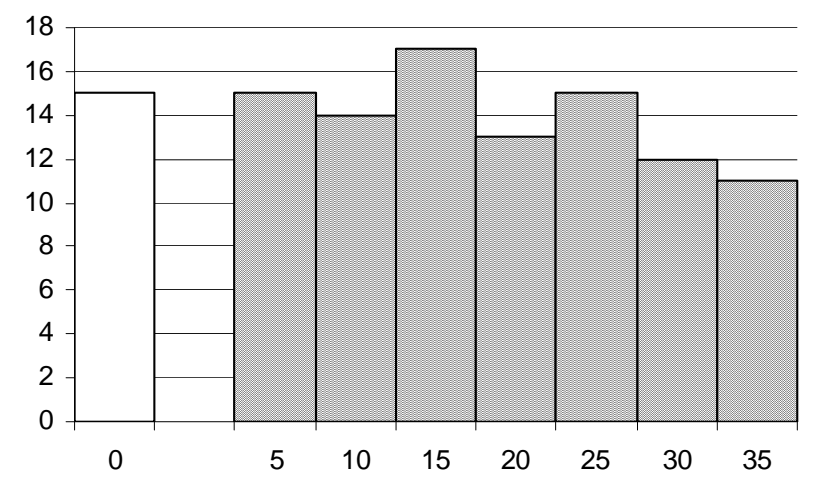

Figure 6. Duration of potentiation of the inhibitory effect of rhythmic stimulation of the reticular nucleus of the thalamus on the background activity of the studied neurons of the ventrolateral nucleus of the thalamus of the cat. Histogram of the values of the above duration measured using the protocol described in the text and in the legend to Figure 6. Abscissa) Duration, min; ordinate) number of neurons with the respective duration of potentiation. Number of the neurons with no or weak potentiation is shown at the left from the ordinate. was several times greater than that at single-pulse TRN stimulations (Figure 7(b)). After this, the TRN-induced effects were tested by single stimulations of this nucleus. In this case, single pulses evoked IPSPs also with an amplitude several times higher than that before conditioning tetanic stimulation (Figure 7(c)). Potentiation of the TRN-induced IPSPs on VL neurons was preserved for 10 - 25 min after termination of tetanic stimulation of the TRN.

\section{Discussion}

\subsection{Interactions between TRN and Thalamic Relay Nuclei Neurons}

Relationships between neurons of the TRN and specific nuclei of the thalamus were studied. Under the influence of stimulation of the TRN unit activity in the thalamic relay nuclei was found to be considerably modulated. Cases of the appearance of IPSPs (possibly of monosynaptic nature), evoked by stimulation of TRN, in neurons of the VL and LGB are described. During simultaneous recording of unit activity in TRN and VL or LGB by means of two electrodes interaction of several types was found: first, inhibition of discharges of VL or LGB neurons accompanied by excitation of TRN neurons; second, alternation of excitation-inhibition in neuron pairs in TRN and VL or LGB during low-frequency afferent or cortical stimulation. In this case excitation of TRN neurons is associated with inhibition of VL or LGB neurons.

The results are in agreement with those of early investtigations by other workers who found that TRN neurons have an inhibitory action on neurons in other parts of the thalamus. Purpura and Cohen [16], for example, first showed that stimulation of the medial thalamus evoked high-frequency discharges of TRN neurons. When recording unit activity in the VL, these workers found deep inhibition of such activity during stimulation of the same kind. A similar result was obtained previously [17] during stimulation of the medial thalamus - the latent periods of evoked high-frequency discharges of TRN neurons were much shorter than latent periods of the corresponding IPSPs of neurons in the VL neurons.

The experimental results showed that after intravenous injections of small doses of pentobarbital the appearance of spindle activity in the EEG was accompanied by activation of most TRN neurons; this was manifested as the appearance of high-frequency grouped (52.5\%) or highfrequency prolonged continuous $(30 \%)$ discharges throughout the period of recording of spindles. Generation of high-frequency discharges by TRN neurons could coincide with an inhibitory pause in activity of VL neurons, and on that basis it might be supposed that inhibition of activity of VL neurons was caused by discharges from TRN neurons. 
a
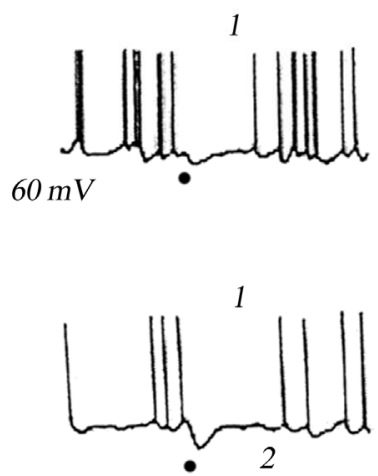

2

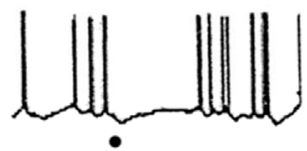

c

2

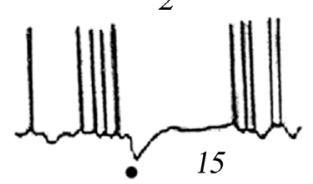

$b$

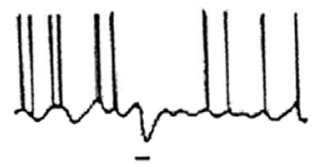

3

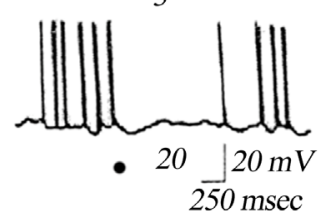

Figure 7. Potentiation of inhibitory postsynaptic effects in a neuron of the VL of the thalamus of the cat after rhythmic stimulation of the TRN. Intracelullar recording from the VL neuron; the membrane potential, $\mathrm{mV}$, is shown at the left. (a) 1 , 2) Effects of single stimulations of the TRN; (b) Effect of the 10th stimulation of the TRN by a series of 10 stimuli of the same intensity as in panel $\mathrm{A}$, applied at a $50 \mathrm{sec}^{-1}$ frequency; interseries intervals were $10 \mathrm{sec}$; (c), 1 - 3, Effects of single stimulations of the TRN with the same intensity applied with different intervals (shown below the traces, min) after termination of rhythmic stimulations.

What are the mechanisms of the increased activity of TRN neurons?

General anesthetics are known $[18,19]$ to inhibit synaptic transmission at all levels in the central nervous system. It is claimed [20] that barbiturates reduced mediator release from presynaptic terminals. In the initial stage of action of barbiturates or when they are given in subnarcotic doses, depression of unit activity manifested by the appearance of periods of silence between separate neuronal discharges. It has been shown that postsynaptic inhibition is intensified at this time. It has been also shown that tha duration of inhibition of spinal motoneurons is increased after injection of barbiturates [21], and that the granule cells of the olfactory bulb [22], Purkinje cells of the cerebellum [23], and neurons of the neocortex [24] are inhibited.

Desynchronization of the EEG, caused by stimulation of mesencephalic reticular formation (MRF), is known to be accompanied by facilitation of the transmission of excitation through thalamic nuclei. Intracellular studies [25] have shown that this facilitation arises because of weakening of IPSPs. Facilitation of transmission of excitation under the influence of stimulation of MRF may thus be result of disinhibition of the neurons which inhibit sensory transmission through the thalamus. It is claimed that inhibition of TRN neurons evoked by stimulation of MRF is cholinergic in nature. It has been shown [26] that inhibitory responses of the same TRN neurons develop both during stimulation of MRF and under the influence of iontophoretic application of acetylcholine. On the other hand, it has been shown that cholinergic synapses are particularly sensitive to barbiturates: their activity is inhibited by barbiturates. It can therefore be postulated that injection of barbiturates will block choliergic axon terminals of MRF neurons entering TRN [27], as well as blocking unit activity at the MRF level; TRN neurons are released from the control of MRF and generate high-frequency discharges, which are characteristic of TRN neurons. As a result of this, TRN neurons have an inhibitory action on thalamic relay neurons.

\subsection{A Possible Mechanism of Blocking of Limbic Motor Seizure Reactions}

Our experiments support the hypothesis that the activity of inhibitory neurons of the TRN is potentiated in the course of stimulation of this structure. This effect is longlasting, and inhibition of generalization of the seizures can be based on this phenomenon.

Long-lasting plastic changes in the efficacy of synaptic transmission were earlier studies in most cases in excitatory synaptic connections in the central nervous system. Analogous studies of the inhibitory synaptic transmission are rather less numerous despite the fact that such a phenomenon per se (potentiation in inhibitory central synapses) was demonstrated long ago by Sherrington [28]. The respective effects were found in neuronal systems of the visual [29] and motor [30] neocortical areas, rostral nucleus of the solitary tract [31], and in Mauthner medullary neurons [32].

Our findings, of course, do not allow us to propose strict single-valued conclusions on synaptic mechanisms of blocking of motor seizures provided by stimulation of the TRN. At the same time, results of the above-described experiments allow us to believe that such a stimulation induces long-lasting plastic modifications in GABAergic synapses formed by axons of the TRN neu- 
rons, in particular, in synapses on neurons of the relay thalamic structures.

The stimulation of the profound cerebral structures has been extensively used in attempts to influence pathological central phenomena both in experiments and clinics. It is obvious that such stimulation excites all neuronal elements (axon, axon terminals and/or cell somata), which are localized at a sufficiently short distance from the electrode. As is known, the excitability of the axons and axon terminals is in general higher than that of the neuronal somata [33]. Neurons of the TRN are supplied by terminals of axon collaterals of both cortico-subcortical and subcortico-cortical origins; thus, stimulation of the TRN inevitably results in activation of all these elements of the nucleus. In any case, a significant proportion of the above axon collaterals are glutamatergic, and their activation should exert a powerful excitatory effect on the TRN neurons. This is why stimulation of the TRN will evoke not only direct activation of neurons of this nucleus, but also their trans-synaptic activation via glutamatergic axon terminals of a cortico-thalamic or a thalamo-cortical origin.

Our experiments have shown that stimulation of the TRN effectively suppresses limbic motor seizures. This suppression is obviously related to the process of potentiation in GABAergic neurons of the TRN. Thus, stimulation of the TRN appears to be a rather valuable methodical tool that can open up prospects in the development of new anticonvulsive strategies in the treatment of temporal lobe epilepsy.

\section{Conclusions}

The data generated in this study indicated: 1) The stimulation of the TRN evoked monosynaptic IPSPs in the thalamic relay neurons; 2) Cortical single and/or rhythmmic stimulation evoked axcitation of the TRN neurons and inhibition of the thalamic relay neurons; 3) After injection of pentobarbital excitation of the TRN neurons corresponded to a period of silence of the thalamic relay neurons; 4) The activity of TRN neurons was significantly enhanced during the silence within the seizure episodes; 5) This suppresion is obviously related to the process of potentiation in GABAergic synapses of thalamo-cortical neuronal networks.

In addition, our data raise the possibility that stimulation of TRN might be of value as a new anticonvulsive approach in patients with intractable temporal lobe epilepsy.

\section{REFERENCES}

[1] R. Davis, "Cerebellar Stimulation for Cerebral Palsy Spastisity, Function and Seizures," Archive of Medical Research, Vol. 31, No. 2, 2000, pp. 290-299.
doi:10.1016/S0188-4409(00)00065-5

[2] G. Weiss, J. Lewis and R. Jimenes-Riverac, "Antikindlig Effects of Locus Coeruleus Stimulation: Mediation by Ascending Noradrenergic Projections," Experimental Neurology, Vol. 108, No. 1, 1990, pp. 136-140. doi:10.1016/0014-4886(90)90020-S

[3] V. Magdaleno-Madrigal, A. Valdés-Cruz, D. MartinezVargas, et al., "Effect of Electrical Stimulation of the Solitary Tract on the Development of Electrical Kindling in the Cat," Epilepsia, Vol. 43, No. 9, 2002, pp. 964-969. doi:10.1046/j.1528-1157.2002.05702.x

[4] F. Velasco, M. Velasco and A. Velasco, "Effect of Chronic Electrical Stimulation of the Centromedian Thalamic Nuclei on Various Intractable Seizure Patyerns: 1. Clinical Seizures and Paroxysmal EEG Activity," Epilepsia, Vol. 34, No. 6, 1993, pp. 1052-1064. doi:10.1111/j.1528-1157.1993.tb02134.X

[5] S. Chanel, M. Westerveld and S. Spenser, "Long-Term Outcome of Vagus Nerve Stimulation for Refractory Partial Epilepsy," Epilepsy and Behavior, Vol. 4, No. 3, 2003, pp. 302-309. doi:10.1016/S1525-5050(03)00109-4

[6] S. Mraovitch and Y. Calando, "Interactions between Limbic, Thalamo-Striatal-Cortical and Central Autonomic Pathways during Epileptic Seizure Progression," Journal of Comparative Neurology, Vol. 411, No. 1, 1999, pp. 145-161. doi:10.1002/(SICI)1096-9861(19990816)411:1<145::AID -CNE11>3.0.CO;2-1

[7] G. Avanzini, M. de Curtis, C. Marescaux F. Panzica, R. Spreafico and M. Vergnes, "Role of the Thalamic Reticular Nucleus in the Generation of Rhythmic ThalamoCortical Activities Subserving Spike and Waves," Journal of Neural Transmition, Vol. 35, 1992, pp. 85-95.

[8] D. Contreras and M. Steriade, "Cellular Basis of EEG Slow Rhythms: A Study of Dynamic Corticothalamic Relationships," Journal of Neuroscience, Vol. 15, No. 1, 1995, pp. 604-622.

[9] C. Cox, J. Huguenard and D. Prince, "Nucleus Reticularis Neurons Mediate Diverse Inhibitory Effects in Thalamus," Proceedings of the National Academy of Sciences of the United States of America, Vol. 94, No. 16, 1997, pp. 88548859. doi:10.1073/pnas.94.16.8854

[10] C. Houser, J. Vaughn, R. Barber and E. Roberts, "GABA Neurons Are the Major Cell Type of the Nucleus Reticularis Thalami," Brain Research, Vol. 200, No. 2-3, 1980, pp. 341-354. doi:10.1016/0006-8993(80)90925-7

[11] U. Kim, M. Sanchez-Vives and D. McCormick, "Functional Dynamics of GABA-Ergic Inhibition in the Thalamus," Science, Vol. 278, No. 5335, 1997, pp. 130-134. doi:10.1126/science.278.5335.130

[12] D. McCormick, "Neurotransmitter Actions in the Thalamus and Cerebral Cortex and Their Role in Neuromodulation of Thalamocortical Activity," Progress in Neurobiology, Vol. 39, No. 4, 1992, pp. 337-388. doi:10.1016/0301-0082(92)90012-4

[13] Z. Nanobashvili, T. Chachua, A. Nanobashvili, I. Bilanishvili and O. Lindvall and Z. Kokaia, "Suppression of Limbic Motor Seizures by Electrical Stimulation in Thalamic Reticular Nucleus," Experimental Neurology, Vol. 181, No. 1, 2003, pp. 224-230. 
doi:10.1016/S0014-4886(03)00045-1

[14] M. Steriade, "Grouping of Brain Rhythms in Corticothalamic Systems," Neuroscience, Vol. 137, No. 4, 2006, pp. 1087-1106.

[15] J. McNamara, D. Bonhous and C. Shin, "The Kindling Model of Epilepsy," In: P. Schwartzkroin, Ed., Epilepsy: Models, Mechanisms and Concepts, Cambridge University Press, Cambridge. 1993, pp. 27-47. doi:10.1017/CBO9780511663314.003

[16] D. Purpura and B. Cohen, "Intracellular Recording from Thalamic Neurons during Recruiting Responses," Journal of Neurophysiology, Vol. 25, No. 5, 1962, pp. 621-630.

[17] T. Frigiesi, "Intracellular Recordings from Neurons in Dorsolateral Thalamic Reticular Nucleus during Capsular, Basal Ganglia and Midline Thalamic Stimulation," Brain Research, Vol. 48, No. 1972, pp. 157-164. doi:10.1016/0006-8993(72)90176-X

[18] S. David, S. Muruyama, X. Machne and K. Unna, "Evidence Supporting Cholinergic Transmission at the Lateral Geniculate Body of the Cat," International Journal of Neuropharmacology, Vol. 2, No. 2-3, 1963, pp. 113-119. doi:10.1016/0028-3908(63)90014-5

[19] I. Schlag, "A Study of the Action of Nembutal on Diencephalon and Mesencephalic Unit Activity," Archive of International Physiology and Biochemistry, Vol. 64, No. 3, 1956, pp. 470-477. doi:10.3109/13813455609150221

[20] C. Richards, "On the Mechanisms of Barbiturate Anaesthesia," Journal of Physiology, Vol. 227, No. 3, 1972, pp. 749758.

[21] M. Larsen and M. Major, "The Effect of Hexobarbital on the Duration of the Recurrent IPSP in the Cat Motoneurons," Brain Research, Vol. 21, No. 2, 1970, pp. 309-316. doi:10.1016/0006-8993(70)90377-X

[22] R. Nicol, "The Effects of Anaesthesia on Synaptic Excitation and Inhibition in the Olfactory Bulb," Journal of Physiology, Vol. 233, No. 3, 1972, pp. 803-812.

[23] J. Eccles, D. Faber and H. Taborikova, "The Action of a Parallel Fiber Volley on the Antidromic Invasion of Purkinje Cells of Cat Cerebellum," Brain Research, Vol. 25, No. 2, 1971, pp. 335-342. doi:10.1016/0006-8993(71)90442-2

[24] F. Serkov, I. Yanovski and A. Talnov, "Effect of Pento- barbital, Chloralose, and Urethane on Inhibitory Postsynaptic Potentials of Cortical Neurons," Neurophysiology, Vol. 6, No. 4, 1974, pp. 339-346.

[25] W. Singer and U. Drager, "Postsynaptic Potentials in Relay Neurons of the Cat Lateral Geniculate Nucleus after Stimulation of the Mesencephalic Reticular Formation," Brain Research, Vol. 41, No. 1, 1972, pp. 214-221. doi:10.1016/0006-8993(72)90632-4

[26] R. Dingledine and I. Kelly, "Brain Stem Stimulation and the Acetylcholine Evoked Inhibition of Neurons in the Feline Nucleus Reticularis Thalami," Journal of Physiology, Vol. 271, No. 1, 1977, pp. 135-147.

[27] N. Nauta and G. Koupers, "Some Ascending Pathways in the Brain Stem Reticular Formation," In: H. H. Jasper, L. D. Proctor, R. S. Knighton, W. C. Noshay and R. N. Costello, Eds., Reticular Formation of the Brain, Boston. 1954, pp. 3-30.

[28] C. S. Sherrington, "The Integrative Activity of the Nervous System," Yale University Press, New Haven and London, 1966.

[29] Y. Komatsu, "Age-Dependent Long-Term Potentiation of Inhibitory Synaptic Transmission in Rat Visual Cortex," Journal of Neuroscience, Vol. 14, No. 11, 1994, pp. 64886499.

[30] T. Labakhua, G. Bekaya and V. Okudzhava, "Post-Tetanic Potentiation of Inhibitory Postsynaptic Potentials in the Motor Cortex of the Cat," Doklady Akademii Nauk SSSR, Vol. 259, No. 4, 1981, pp. 1013-1015. (in Russion).

[31] G. Grabauskas and R. Bradly, "Potentiation of GABAergic Synaptic Transmission in the Rostral Nucleus of the Solitary Tract," Neuroscience, Vol. 94, No. 4, 1999, pp. 1173-1182. doi:10.1016/S0306-4522(99)00379-6

[32] H. Korn, Y. Oda and D. Faber, "Long-Term Potentiation of Inhibitory Circuits and Synapses in the Central Nervous System," Proceedings of the National Academy of Sciences of the United States of America, Vol. 89, No. 1, 1992, pp. 440-443. doi:10.1073/pnas.89.1.440

[33] L. Novak and J. Bullier, “Axons, but Not Cell Bodyes, Are Activated by Electrical Stimulation in Cortical Gray Matter 1. Evidence from Chronaxy Measurement," Experimental Brain Research, Vol. 118, No. 4, 1998, pp. 477-488. doi:10.1007/s002210050304 